Research Paper

\title{
Constitutive activation of MEK5 promotes a mesenchymal and migratory cell phenotype in triple negative breast cancer
}

\author{
Margarite D. Matossian ${ }^{1 *}$, Van T. Hoang ${ }^{1 *}$, Hope E. Burks ${ }^{1 *}$, Jacqueline La $^{1 *}$, \\ Steven Elliott ${ }^{1}$, Courtney Brock ${ }^{1}$, Douglas B. Rusch ${ }^{2}$, Aaron Buechlein ${ }^{3}$, Kenneth P. \\ Nephew $^{3}$, Akshita Bhatt ${ }^{4}$, Jane E. Cavanaugh ${ }^{4}$, Patrick T. Flaherty ${ }^{5}$, Bridgette M. \\ Collins-Burow ${ }^{1,6}$, Matthew E. Burow ${ }^{1}$ \\ ${ }^{1}$ Department of Medicine, Division of Hematology and Oncology, Tulane University, New Orleans, LA 70118, USA \\ ${ }^{2}$ Center for Genomics and Bioinformatics, Indiana University, Bloomington, IN 47405, USA \\ ${ }^{3}$ Medical Sciences Program, Indiana University School of Medicine-Bloomington, Bloomington, IN 47405, USA \\ ${ }^{4}$ Department of Pharmacology, Duquesne University School of Pharmacy, Pittsburgh, PA 15282, USA \\ ${ }^{5}$ Department of Medicinal Chemistry, Duquesne University School of Pharmacy, Pittsburgh, PA 15282, USA \\ ${ }^{6}$ Tulane Cancer Center, New Orleans, LA 70112, USA \\ * These authors contributed equally to this work and are shared first authors. \\ Correspondence to: Matthew E. Burow, email: mburow@tulane.edu \\ Keywords: triple negative breast cancer; MEK5; epithelial to mesenchymal transition; cell migration; ERK5 \\ Received: December 13, $2020 \quad$ Accepted: April 26, $2021 \quad$ Published: May 18, 2021
}

Copyright: (๑) 2021 Matossian et al. This is an open access article distributed under the terms of the Creative Commons Attribution License (CC BY 3.0), which permits unrestricted use, distribution, and reproduction in any medium, provided the original author and source are credited.

\section{ABSTRACT}

Triple negative breast cancer (TNBC) is an aggressive subtype of breast cancer with limited targeted therapeutic options. A defining feature of TNBC is the propensity to metastasize and acquire resistance to cytotoxic agents. Mitogen activated protein kinase (MAPK) and extracellular regulated kinase (ERK) signaling pathways have integral roles in cancer development and progression. While MEK5/ ERK5 signaling drives mesenchymal and migratory cell phenotypes in breast cancer, the specific mechanisms underlying these actions remain under-characterized. To elucidate the mechanisms through which MEK5 regulates the mesenchymal and migratory phenotype, we generated stably transfected constitutively active MEK5 (MEK5-ca) TNBC cells. Downstream signaling pathways and candidate targets of MEK5-ca cells were based on RNA sequencing and confirmed using qPCR and Western blot analyses. MEK5 activation drove a mesenchymal cell phenotype independent of cell proliferation effects. Transwell migration assays demonstrated MEK5 activation significantly increased breast cancer cell migration. In this study, we provide supporting evidence that MEK5 functions through FRA-1 to regulate the mesenchymal and migratory phenotype in TNBC.

\section{INTRODUCTION}

Triple negative breast cancer (TNBC) represents a particularly aggressive subtype of breast cancer. Defined by a lack of targetable receptors, treatment options for patients presenting with TNBC are limited to cytotoxic agents, such as anthracyclines and taxanes [1,2]. Although TNBCs are initially responsive to these therapies, the risk of relapse for TNBC patients is much higher than that of women with hormone receptor positive breast cancer, with an overall worse outcome [2]. Heterogeneity within TNBC partially contributes to acquired resistance to cytotoxic agents $[1,2]$. This chemotherapeutic resistance, both primary and acquired, remains a significant challenge in the clinic. Given its clinical importance, there is particular interest in determining new therapeutic targets against chemoresistance, particularly in the context of TNBC, a cancer subtype that lacks receptor- 
based targeted treatments [1-3].

Aberrant MAPK signaling has been broadly shown to mediate chemoresistance in numerous malignancies [47]. Activation of MAPK pathways through either mutation or direct activation promotes expression of cell survival genes and inhibits apoptosis [4, 7]. Although anti-MEK1/2 targeted therapies have been generated, the inevitable development of secondary mutations prevents the effective long-term use of these drugs in treatment regimens [7]. However, the MAP2K5, or MEK5, pathway is less characterized than other MAPK signaling pathways, and may offer an alternative target. Our lab and others have characterized MEK5/ERK5 signaling as a driver of epithelial to mesenchymal transition (EMT), resistance to apoptosis, and cell survival $[8,9]$. Specifically, we have demonstrated that MEK5/ERK5 signaling mediates progression to a mesenchymal and endocrine therapy-resistant phenotype [10] and knockdown of this pathway suppresses growth and metastasis of MDAMB-231 tumors [11]. We have also previously shown that activated ERK5 has elevated expression in breast tumors compared to adjacent normal tissue [10]. With respect to metastasis, activated ERK5 had elevated expression in brain metastases from clinically aggressive breast tumors [12]. The MEK5/ERK5 pathway regulates transcription factors that mediate the EMT phenotype including phosphorylation and increased activation of c-Fos and Fra-1 [8-10, 13, 14]. However, more extensive characterization of this relationship is needed. While Fra-
1 expression activates a mesenchymal phenotype in breast cancer [15], further data is required to further support a direct relationship between MEK5/ERK5 signaling and the Fra-1-driven EMT phenotype in breast cancer.

EMT plays an integral role in regulating various processes crucial to development, progression and recurrence of breast tumors, including metastasis, maintenance of breast cancer stem cells and acquisition of drug resistance [15-20]. In breast cancer cells, MEK5 overexpression promoted a TNF $\alpha$ resistance phenotype $[9,21]$. Activation of the MEK5 pathway has been shown to confer a survival advantage to colon cancer cells when treated with the pyrimidine analog 5 -fluorouracil (5-FU) [22]. In TNBC cells, ERK5 pharmacologic inhibition amplified anti-cancer effects of cytotoxic chemotherapies Taxotere, vinorelbine and cisplatin [23]. Conversely, ERK 5 mRNA expression is associated with poor regression free survival in breast cancer patients receiving chemotherapy [24].

Characterizing signaling pathways that mediate EMT in breast cancer biology provides a promising avenue for novel therapies. Given the clinical relevance and significant regulatory roles of the MEK5/ERK5 signaling pathway in breast cancer, it is a promising therapeutic target $[22,25,26]$. Here, we present supporting evidence that constitutive activation of MEK5 drives a mesenchymal and migratory TNBC phenotype in TNBC cells.
A

Kaplan Meier gene expression RNAseq - IlluminaHiSeq

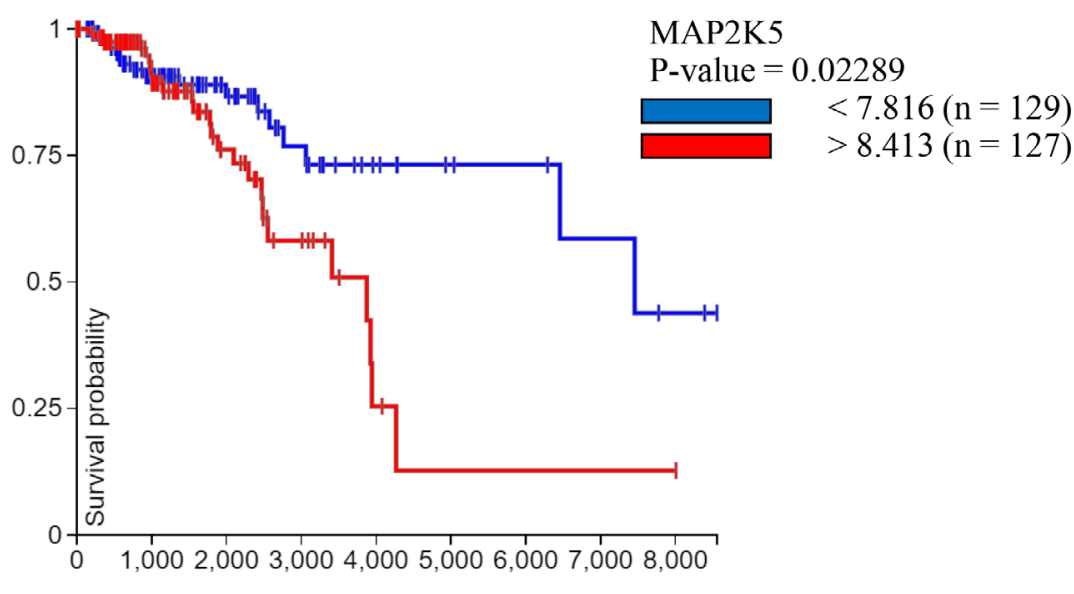

B MAP2K5

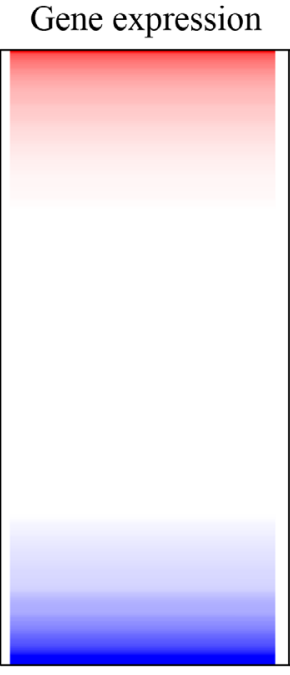

TFAC30

Signature

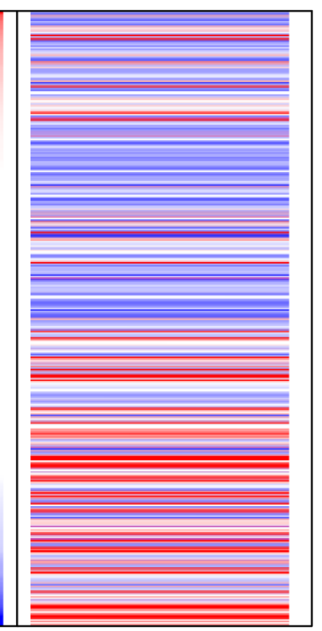

Figure 1: MEK5 (MAP2K5) gene expression associated with worse overall survival. (A) Kaplan-Meier survival plot of MEK5 (MAP2K5) gene expression extrapolated from available RNAseq data (IlluminaHiSeq) demonstrating higher MEK5 expression is associated with worse survival probability. (B) MEK5 (MAP2K5) gene expression is inversely correlated to the TFAC30 gene signature for complete pathologic response to cytotoxic drug therapies. Data was obtained from (A) the Kaplan-Meier plotter and (B) the TCGA databases. 


\section{RESULTS}

\section{MEK5 expression is associated with worse patient survival}

First, we performed a Kaplan Meier gene expression analysis with MEK5 (MAP2K5) to investigate effects of MEK5/ERK5 in a clinical setting. We observed that increased MEK5 expression was associated with reduced patient survival probability in breast cancer (Figure 1A). Metastasis is closely associated with therapeutic response and survival in TNBC. We examined the patterns of MEK5 expression in patient data using the Xena browser and TCGA cancer browser. The TFAC 30 gene signature was generated by Hess et al., and lists 30 genes whose gene expression profile is predictive of complete pathologic response to chemotherapy treatment in breast cancer [27]. This signature is high in the basal subtype and ER negative patient samples. This gene signature is as follows: E2F3 + MELK + RRM2 + BTG3 - CTNND2 GAMT - METRN - ERBB4 - ZNF552 - CA12 - KDM4B - NKAIN1 - SCUBE2 - KIAA1467 - MAPT - FLJ10916 - BECN1 - RAMP1 - GFRA1 - IGFBP4 - FGFR1OP -
MDM2 - KIF3A - AMFR - MED13L - BBS4. Elevated MAP2K5 gene expression in breast cancer patients across all subtypes inversely correlated with the TFAC30 gene signature (Figure 1B). Together, these data are in line with previous studies which have investigated the MEK5 signaling axis in breast cancer outcomes using the Kaplan Meier analyses and strengthen the importance of MEK5 signaling in breast cancer progression and outcomes.

\section{MEK5 drives EMT and cell migration}

We next sought to identify and characterize the pathways and processes by which MEK5 exerts protumorigenic effects in TNBC. To address this, we employed two basal subtype TNBC cell lines (MDAMB-231, Hs-578T). While these TNBC cells are categorized in the same molecular subtypes, they have distinct morphological and mutational profiles and both cell lines were included to account for cell type-specific differences. A constitutively active MEK5 expression construct (MEK5-ca) was generated in MDA-MB-231 and Hs-578T cells. Stable transfection of MEK5 overactivation was confirmed on a transcript (Figure 2A) and protein level (Figure 2B). Global transcriptome analysis of
A

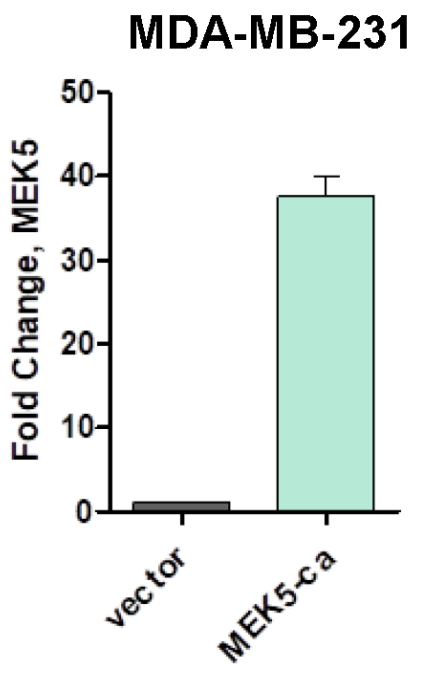

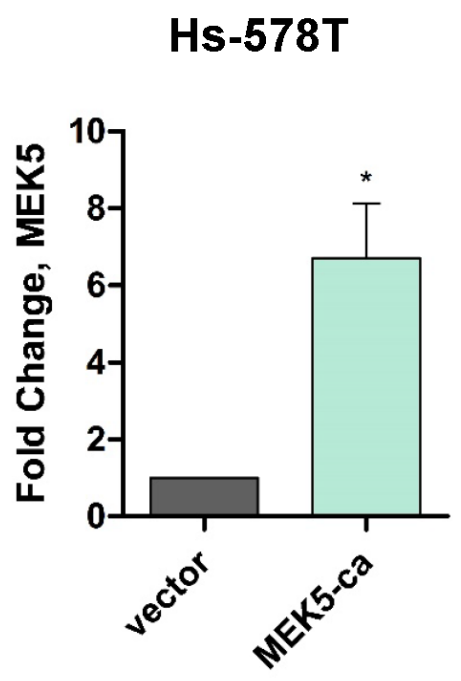

$\mathbf{B}$
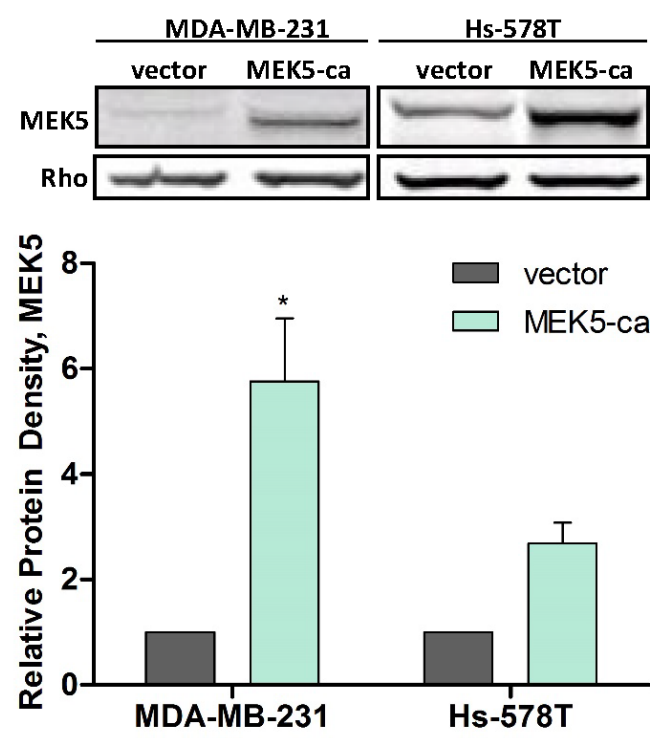

Figure 2: Confirmation of MEK5-ca cells. MDA-MB-231 and Hs-578T cells were transfected with vector or MEK5DD plasmid. Cells were treated with selectable marker (puromycin). Viable colonies were cloned and pooled for analysis. qPCR was performed on (A) MDA-MB-231- and Hs-578T-vector and -MEK5-ca cells for MEK5 expression. (B) Total protein was extracted from TNBC-MEK5-ca cells and western blot was performed for total MEK5 expression. Rho-GDI $\alpha$ served as a loading control. Bars represent normalized protein density \pm SEM and vector-control cells set to $1, \mathrm{n} \geq 3$. ${ }^{*} \mathrm{p}<0.05 ;{ }^{* *} \mathrm{p}<0.01$. 
these MDA-MB-231-MEK5-ca cells was performed and identified genes that were differentially expressed $(\mathrm{FC}<$ 2, $\mathrm{p}<0.05)$ in the MEK5-ca expressing cells compared to the vector control. Gene Set Expression Analysis (GSEA) revealed a predicted increase in activation of the epithelial to mesenchymal transition (EMT) pathway in MEK5-ca cells when compared to control (Figure 3A). Consistent with these RNA-seq data, we observed an upregulation in expression of EMT associated factors FRA-1, SNAI1, MMP1, MMP2, and IL-8 in MDA-MB231-caMEK5 cells compared to vector control (Figure 3B). Additionally, MEK5-ca increased expression of downstream MEK pathway members (MEF2A, MEF2C) and mesenchymal genes (CDH2, MMP2) (Figure 3C). MEK5-ca significantly downregulated the epithelial marker CDH1 gene expression (Figure 3C). Moreover, MEK5-ca cells showed greater migration potential, 3.25fold ( $\mathrm{p}<0.01$ ) of MDA-MB-231- and 1.24-fold ( $\mathrm{p}<$ 0.05 ) of Hs-578T-vector cells (Figure 3D). These findings suggest that constitutive activation of MEK5 regulates the EMT/migration axis.

Our group, and others, have proposed FRA1 to be a key downstream target of the MEK5/ERK5 pathway that regulates the EMT phenotype. We further characterized this relationship and showed that while constitutive activation of MEK5 does not increase total FRA-1, p-FRA-1 protein expression was significantly increased (Figure 4A, B). Furthermore, it was determined in both Hs-578T and MDA-MB-231 cells that MEK5ca regulated the FRA-1 response to pan-MEK inhibition (MEK1/2 and MEK5 inhibition) with SC-151 (Figure 4C, D; Supplementary Figure 2). These data demonstrate that MEK5 functions through FRA-1 signaling activation.

\section{MEK5 does not affect cell proliferation}

Gene expression analysis implicated MEK5 in cell growth and proliferation in both IPA disease and function analysis, as well as in GSEA canonical pathway analyses (Figure 5A, B). We next examined whether MEK5 activation promotes chemoresistance through increases in cell proliferation. We used Ki-67 staining to identify changes in proliferative capacity in the MEK5ca cells compared with control in MDA-MB-231 cells. Contrary to expectation, we found that increases in MEK5 signaling alone did not induce increased proliferation in TNBC cells (Figure 5C, D). However, these cells are
A

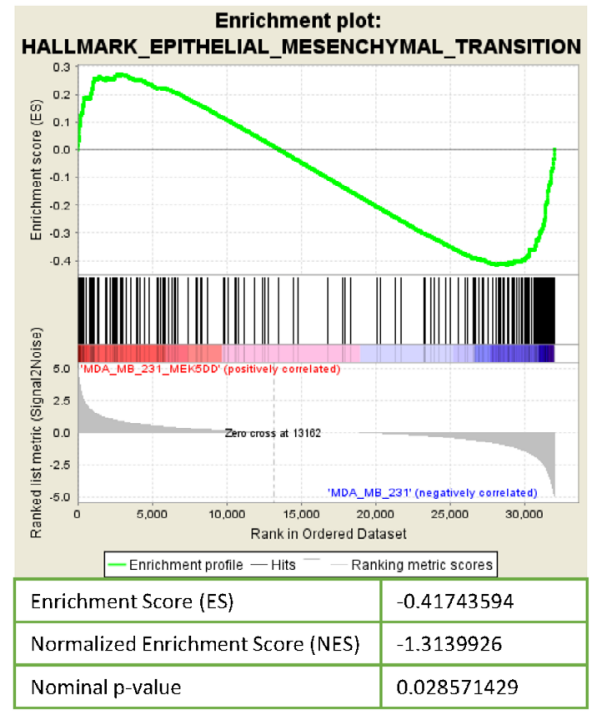

B
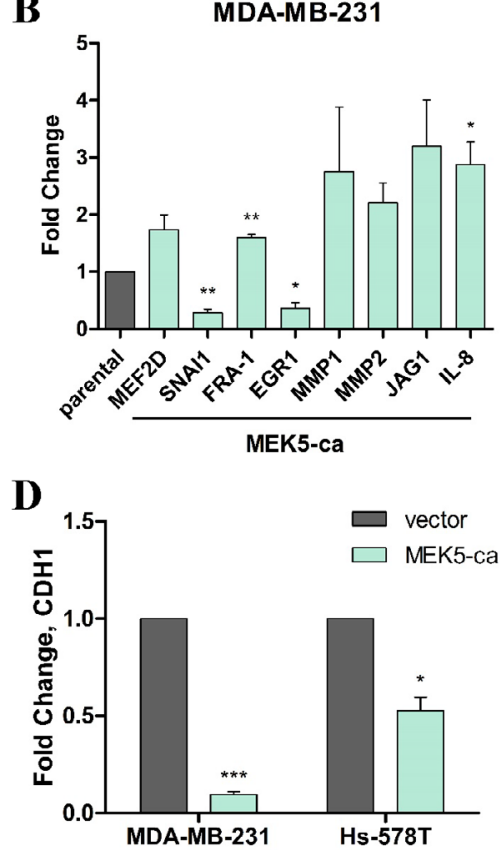

C

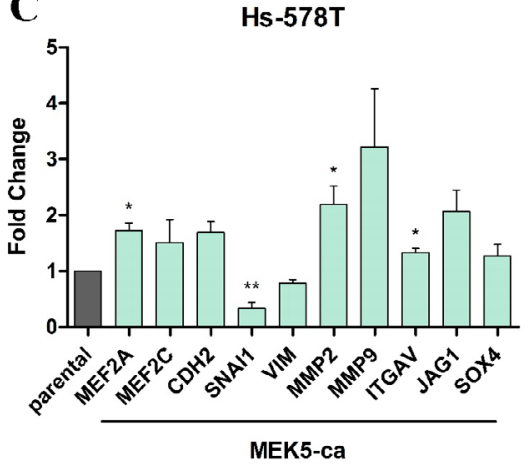

$\mathbf{E}$

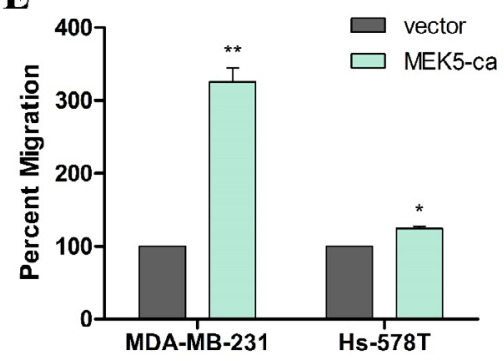

Figure 3: Constitutive activation of MEK5 downregulates CDH1 expression and enhances TNBC cell migration. (A) GSEA analysis of RNA sequencing of MDA-MB-231-MEK5-ca cells compared to parental controls demonstrating upregulation of EMT genes in MEK5-ca cells. qPCR for EMT markers in (B) MDA-MB-231 or (C) Hs-578T-vector and -MEK5-ca cells (C) Western blot of the epithelial marker CDH1 in MEK5-ca cells. (D) Transwell migration assay for MDA-MB-231- or Hs-578T-parental and -ERK5-ko cells. After 24 hours, migrated cells were fixed, stained with crystal violet, and quantified. Bars represent average number of migrated cells normalized to parental cells (set to $100 \%$ ) \pm SEM of triplicate experiments. $* \mathrm{p}<0.05 ; * * \mathrm{p}<0.01 ; * * * \mathrm{p}<0.001$. 

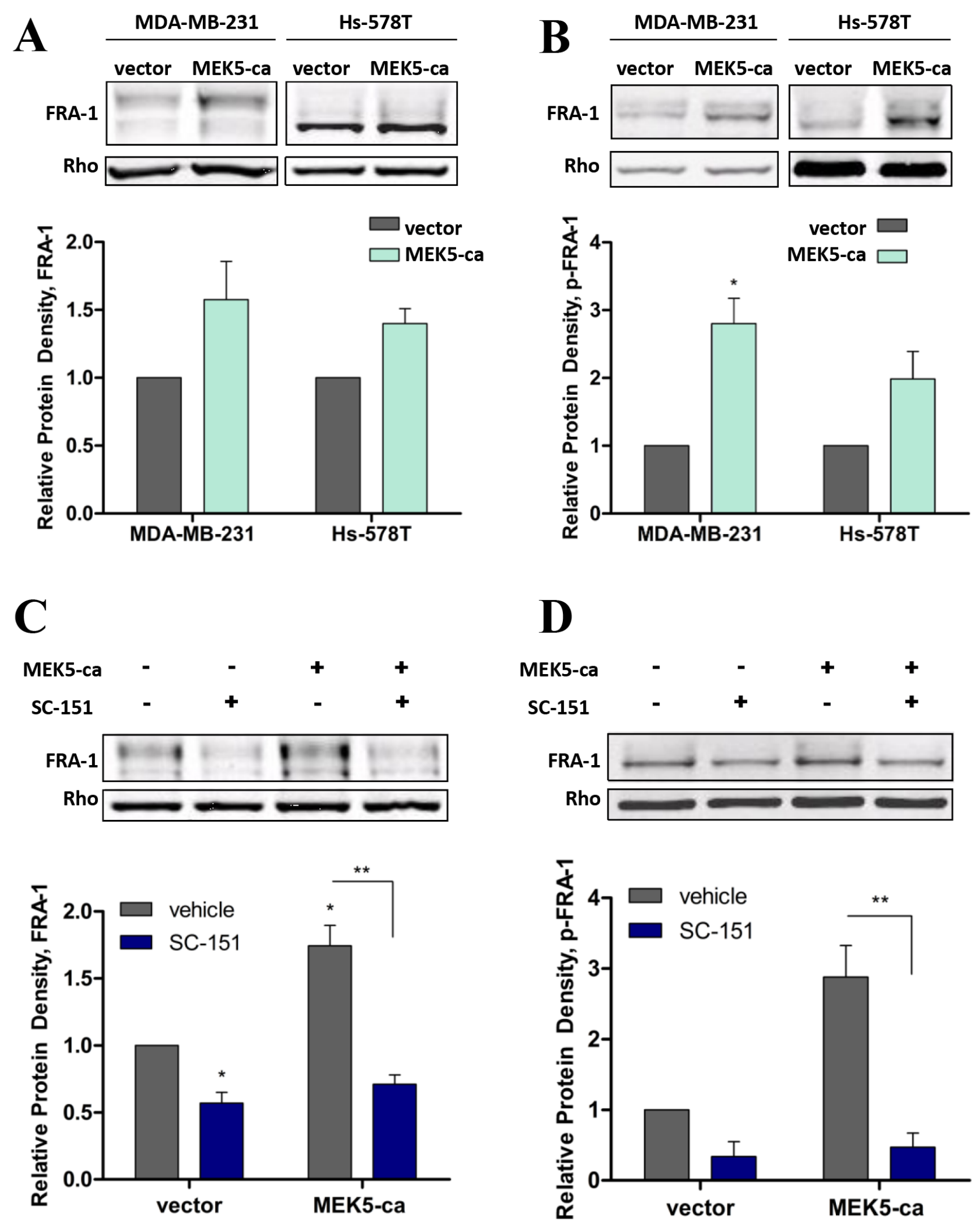

Figure 4: MEK5 regulation of FRA-1 expression. Western blot analysis of (A) FRA-1 and (B) p-FRA-1 expression in TNBC cells, $\mathrm{n} \geq 2$. Western blot analysis for (C) FRA-1 and (D) p-FRA-1 expression in Hs-578T-parental and -MEK5-ca cells treated with vehicle or SC-151 $(1 \mu \mathrm{M})$ for 24 hours, $\mathrm{n}=3$. * $\mathrm{p}<0.05 ; * \mathrm{p}<0.01$. 
highly proliferative at baseline levels. We predicted that the activation of these cell cycle pathways might confer resistance to DNA damaging chemotherapeutics by way of maintaining active cell proliferation. We hypothesized that MEK5 does not confer increased proliferation but instead allows cells to sustain their proliferative phenotype even in presence of chemotherapeutic agents. This sustained proliferation may contribute to chemotherapeutic resistance in high MEK5 expressing TNBC tumors.

\section{DISCUSSION}

Given the diverse and integral regulatory functions of MAPK members and downstream targets in breast cancer progression and resistance, MAPK signaling pathways are promising additions to adjuvant chemotherapy regimens. Specifically, the MEK5/ERK5 pathway has emerged as a promising novel therapeutic target for breast cancer, as this signaling pathway regulates processes integral to breast cancer, including initiation, progression, metastasis, and drug resistance. Increased EMT and acquisition of mesenchymal features drives many of these processes, through maintenance of a cancer stem cell-like populations, promotion of cell motility and ultimately metastasis, and resistance to cytotoxic anticancer drugs. MEK5 signaling has been shown to promote EMT, cell survival, and evasion of apoptosis mechanisms linked to adaptive resistance [28-32]. The efficacy of cytotoxic agents used in chemotherapy, the standard-of-care for various cancer types, is mitigated by activation of signaling pathways, such as MEK5, that confer drug resistance [29]. In basal-like breast cancer subtypes, overexpression of MEK5 in conjunction with ERK5 was associated with poor relapse- and metastasisfree survival in patients who received chemotherapy compared to patients not treated with chemotherapy, which suggests that MEK5-ERK5 expression could serve as a predictive marker for patient benefit from systemic treatments in the ER-negative breast cancer setting [24]. Moreover, in MDA-MB-231 cells ERK5 inhibition by TG02 augmented anti-cancer effects of chemotherapeutic agents conventionally used in TNBC treatment, including taxotere, vinorelbine, and cisplatin [22]. These results support the role of MEK5 signaling in regulation of survival and apoptosis and implicate MEK5 pathway involvement in chemoresistance [21]. Notably, our data suggested that MEK5 activation upregulated cell cycle pathways based on RNA sequencing analyses, but cell
A

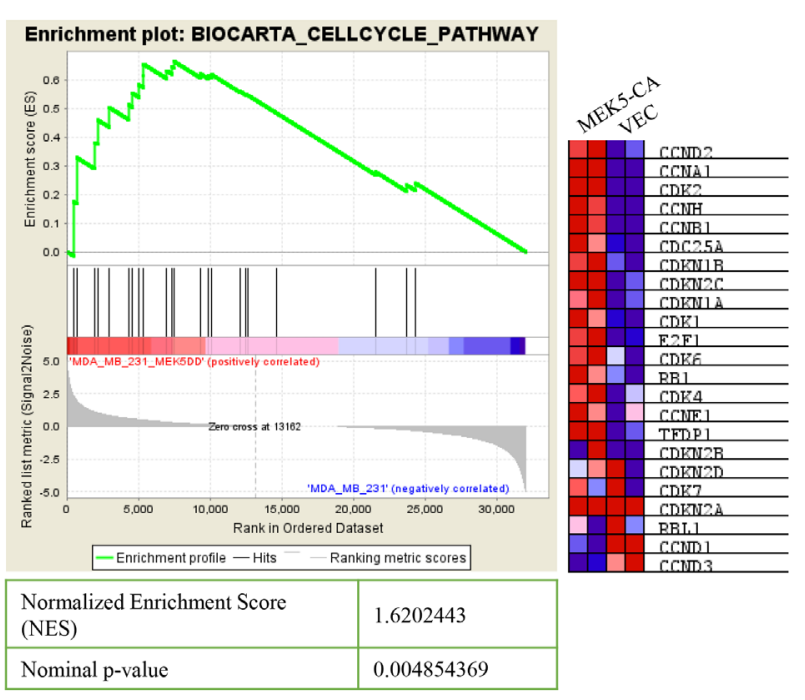

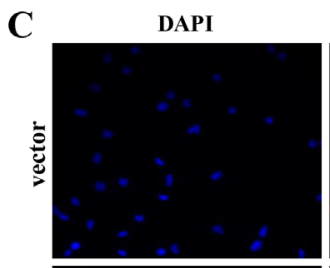
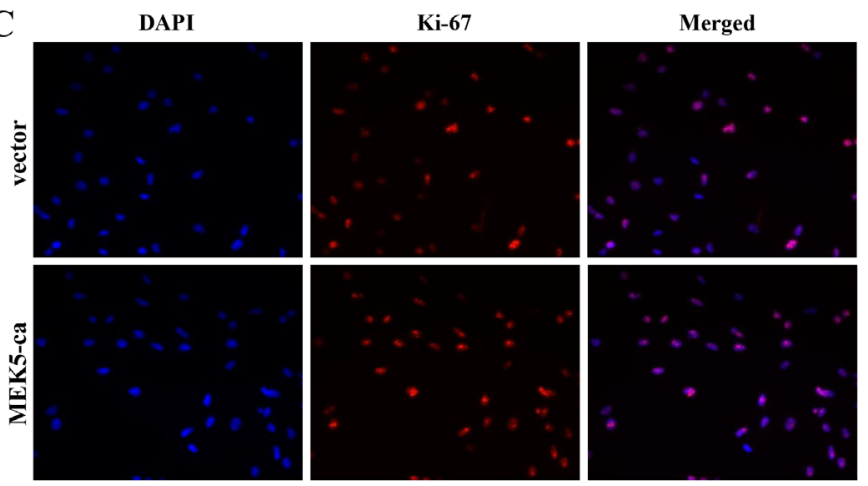

D

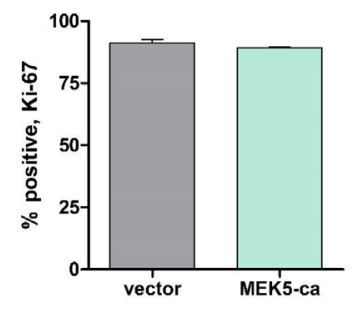

Figure 5: MEK5-ca upregulates cell cycle pathways but does not affect cell proliferation. (A) GSEA analysis of RNA sequencing of MDA-MB-231-MEK5-ca cells compared to parental controls demonstrating upregulation of (B) cell cycle related genes in MEK5-ca cells. (C) IF staining of Ki-67 in MDA-MB-231-vector and -MEK5-ca cells, viewed at 200x. (D) Representative images were taken per well and percentage of Ki-67-positive cells relative to total (DAPI-positive) cells was calculated. Bars represent mean \% of Ki-67 positive cells \pm SEM of triplicate experiments. 
proliferation was not dramatically affected by MEK5/ ERK5. These data are consistent with published data that have found MEK5-ERK5 signaling regulate cell cycle progression, notably by mediating G1/S transition through regulating cyclin D1 expression and through regulation of $\mathrm{G} 2 / \mathrm{M}$ transition and is required for mitotic entry [33]. However, our data shows that MEK5 activation alone is not sufficient to increase proliferation in MDA-MB-231 cells. This supports the hypothesis that pathways affected by MEK 5 activation may be cell type specific. This hypothesis is further supported by our findings that while constitutive activation of MEK5 increased migration of both TNBC cell lines, there were differences in the level of response amongst transfected Hs-578T and MDAMB-231 cells.

Interestingly, when MEK5-ca cells were treated with the MEK1/2/5 inhibitor SC-1-151. Although previously observed cellular activity of SC-151 is consistent with computationally anticipated type III MEK5 inhibitor affinity [34, 35], observations in this report examining activity of SC-1-151 against constitutively active mutant MEK5DD suggest that there may be additional interactions beyond type III MEK5 interactions. Classically, type III inhibitors displace the c-helix and prevent MEK activation via dual phosphorylation on the TEY motif. There has, however, been an increasing awareness [36-41] that type-III kinase binders may induce additional allosteric modification beyond displacement of the C-helix. There also exists the possibility that SC1-151 may have interactions at other MEK5 allosteric sites, may modify a MEK5 protein/protein interaction, or have activity at a yet uncharacterized protein. Further experiments are necessary to establish interactions at the molecular level through clinical relevance to fully evaluate these observations. These findings demonstrate the need for a greater understanding of MEK5 inhibition; assays contributing to this understanding are actively in development in our collaborative laboratory groups.

While a link between activated MEK5/ERK5 signaling and EMT has been demonstrated [8, 9, 28, 42], specific downstream substrates that are responsible for this activity remains understudied. EMT transcription factors (EMT-TF), including the SNAIL, TWIST, and ZEB families, as well as c-Fos and Fra-1 promote the acquisition of a mesenchymal phenotype. EMT-TFs regulate cell plasticity and are involved in many stages of cancer progression such as initiation, primary growth, invasion, dissemination, metastasis, and drug resistance [43-45]. Within subsets of metastatic breast cancer, EMTTFs are associated with poor prognoses and increased risk of metastatic outcomes [44, 45]. In this study, we found the MEK5/ERK5 signaling activates the EMT-TF Fra-1, which is responsible for driving a mesenchymal and migratory cell phenotype. These findings provide supportive evidence consistent with prior studies that activation of MEK5 upregulates breast cancer cell proliferation, migration and promotes the mesenchymal phenotype through FRA-1 signaling activation.

\section{MATERIALS AND METHODS}

\section{Reagents and cell culture}

MDA-MB-231 and Hs-578T cell lines, both categorized as mesenchymal-like TNBC cells of the basal intrinsic subtype with different molecular characteristics [46], were acquired from American Type Culture Collection. Both cell lines were cultured in Dulbecco's Modified Eagle Medium (DMEM; pH 7.4; Invitrogen, Carlsbad, CA) supplemented with 10\% Fetal Bovine Serum (FBS; Hyclone, Salt Lake City, UT), 1\% nonessential amino acids, minimal essential amino acids, sodium pyruvate, antibiotic/anti-mycotic and insulin under mycoplasma-free conditions at $37^{\circ} \mathrm{C}$ in humidified 5\% CO2 and 95\% air. Dimethylsulfoxide (DMSO) was purchased from Fisher Scientific (Waltham, MA). Dosing for SC-1-151, which inhibits MEK1, MEK2 and MEK5, kindly provided by Patrick Flaherty (Duquesne University, Pittsburgh, PA), was $1 \mu \mathrm{M}$ for in vitro studies unless otherwise indicated. While cells were maintained in $10 \%$ FBS-containing DMEM as described above, for drug treatment experiments cells were placed in low serum media, or phenol-free media supplemented with 5\% charcoal-stripped FBS, Glutamax (ThermoFisher Scientific, \#35050079), non-essential amino acids, minimal essential amino acids, and penicillinstreptomycin.

\section{Generation of constitutively active MEK5 TNBC cells}

The constitutively active pcDNA3-MEK5(DD) (MEK5-ca) expression plasmid, graciously donated by Marcus Buschbeck (Max-Planck-Institute of Biochemistry, Martinsried, Germany), was produced by site-directed mutagenesis replacing S311 and T315 by aspartate (D). TNBC cells were plated in $10 \mathrm{~cm}$ dishes and allowed to adhere overnight at $37^{\circ} \mathrm{C}$. Cells were transfected with 5 $\mu \mathrm{g}$ of plasmid in $300 \mu \mathrm{L}$ Opti-MEM. Transfection was accomplished using $15 \mu \mathrm{L}$ Attractene per manufacturer's instructions (Qiagen, Valencia, CA). Media was changed the following day and cells were treated with selectable marker every two days. Once stable cells were obtained, viable colonies were cloned (MDA-MB-231) or pooled (Hs-578T). Stable expression was confirmed by qPCR and Western blot. 


\section{Quantitative polymerase chain reaction (PCR)}

Cells were grown in phenol red-free DMEM supplemented with $5 \%$ charcoal-stripped (CS) fetal bovine serum (5\% CS-DMEM) for 48 hours and treated with compounds. After 24 hours, cells were collected and total RNA was extracted using the Quick RNA Mini Prep Kit in accordance with the manufacturer's protocol (Zymo Research, Irvine, CA). The quality and concentration of RNA were determined spectrophotometrically by absorbance at 260 and $280 \mathrm{~nm}$ using the NanoDrop ND1000. Total RNA $(1 \mu \mathrm{g})$ was reverse-transcribed using the iScript kit (BioRad, Hercules, CA) and qPCR was performed using SYBR-green (Bio-Rad Laboratories, Hercules, CA). Cycle number was normalized to $\beta$-actin and vehicle-treated cells scaled to $1, \mathrm{n}=3$. For patientderived xenografts, RNA was isolated from tumor pieces using QIAzol Lysis Reagent (Qiagen, Valencia, CA) and Quick RNA Mini Prep Kit (Zymo Research, Irvine, CA).

\section{Western blot}

Cells were cultured in 10\% FBS-supplemented DMEM. At confluence or post 24-hour treatment, cells were collected in PBS, pelleted, and lysed with mammalian protein extraction reagent (MPER) supplemented with $1 \%$ protease inhibitor and $1 \%$ phosphatase inhibitors (I/II) (Invitrogen, Grand Isles, NY). Samples were centrifuged at 12,000 RPM for ten minutes at $4^{\circ} \mathrm{C}$ to obtain supernatant containing protein extracts. NanoDrop ND-1000 was used to determine protein concentration of samples by absorbance at 260 and $280 \mathrm{~nm}$. After proteins were heat-denatured at $100^{\circ} \mathrm{C}$ on a heating block, $40 \mu \mathrm{g}$ of protein was loaded per lane on Bis-Tris-nuPAGE gel (Invitrogen, Grand Isles NY). Protein was then transferred to nitrocellulose membranes using iBlot and iBlot transfer stacks per manufacturer's instructions (Invitrogen, Grand Isles, NY). Membrane was incubated at room temperature with $5 \%$ bovine serum albumin (BSA) in 1\% Tris-buffered saline, $0.1 \%$ Tween 20 (TBS-T) for 1 hour to block non-specific binding followed by $4^{\circ} \mathrm{C}$ incubation overnight with primary antibodies (MEK5: anti-rabbit, Santa Cruz, 10795; p-FRA-1 (Ser 265), Cell Signaling Technology, 3880; FRA-1 (D80B4), Cell Signaling Technology, 5281). After three 15-minute washes in 1\% TBS-T, membranes were incubated with appropriate secondary antibodies for at least one hour. IR-tagged secondary antibodies were purchased from LiCor Biosciences (Lincoln, NE) and used at a 1:10,000 dilution in 5\% BSA. Following incubation with secondary antibodies, membranes were washed three times for 15 minutes per wash in 1\% TBS-T, and blots were analyzed by the Odyssey Infrared Imaging
System (LiCor Biosciences). Band density was quantified by LiCor gel imager. Data were normalized to Rho GDI- $\alpha$ (Santa Cruz Biotechnology, Santa Cruz, CA), serving as loading control. Experiments were conducted in triplicate with representative blots shown.

\section{Transwell migration assay}

Cells $\left(2.5 \times 10^{4}\right)$ in $500 \mu \mathrm{L}$ phenol red-free OptiMEM were seeded in the upper chamber of a 24well transwell chamber. 5\% DMEM was used as a chemoattractant in the lower wells. Phenol red-free OptiMEM was used in one well as a negative control to assess basal migration rates. After 24 hours, inner membranes were scrubbed to remove non-migrated cells. Cells on the outer membranes were fixed in formalin and stained with crystal violet. Membranes were excised from the transwell insert and mounted on glass slides. Number of migrated cells were visualized by microscopy and counted. Bars represent percent control migrated cells per 200x field of view \pm standard error of mean (SEM) for triplicate experiments.

\section{Immunofluorescent staining}

Cells were seeded in 96-well plates at a density of 2,000 cells per well. For morphometric analysis, cells were fixed in formalin 3 days after drug treatment and permeabilized with Triton X-100 (Sigma, St. Louis, MO, USA). The cytoskeleton was identified with Alexa Fluor ${ }^{\circledR} 555$ to visualize phalloidin (1:200; Cell Signaling Technologies). Cells were counterstained with DAPI (1:1000; Invitrogen). ApoTome fluorescent images were taken on an inverted microscope (Zeiss, Thornwood, NY) and digitally filtered to obtain optical slices. For Ki-67 analysis, cells were fixed in-well and stained with Alexa Fluor ${ }^{\circledR} 555$ conjugated to Ki-67 (1:200; BD Biosciences). 5 images per well were captured at $400 \mathrm{x}, \mathrm{n}=3$. Results are represented as percent positive Ki-67 staining (red) of total number of cells visualized by DAPI (blue).

\section{Whole genome sequencing and pathway analysis}

MDA-MB-231 transfected cells were extracted for total RNA. Changes in gene expression were determined using next generation sequencing as described [46]. Genes significantly up-regulated in both cell lines were pooled and uploaded into the online pathway interaction database (PID) [http://www.cancer.gov], followed by analysis of significantly down-regulated genes. Based on $-\log (\mathrm{p}$-value) calculated from output data, top regulated pathways were determined. 


\section{Statistical analysis}

Statistical analyses were performed using Graphpad Prism software (Graph-Pad Software, Inc., San Diego, CA). Data were subjected to unpaired Student's t-test, with p-value $<0.05$ considered statistically significant. Studies involving more than two groups were analyzed by one-way analysis of variance (ANOVA) followed by Tukey's post-hoc multiple comparison tests. ${ }^{*}, \mathrm{p}<0.05$; $* *, \mathrm{p}<0.01 ; * * *, \mathrm{p}<0.001$.

\section{AUTHOR CONTRIBUTIONS}

MDM, HEB, VTH wrote and put together the first draft of the manuscript. VTH, SE and HEB generated the stably transfected cells and performed the Western blot, transwell migration and qPCR experiments. MDM performed the cell survival and activity assays for drug resistance. AB, JC, PTF, CB, SE, MEB, BC-B revised the manuscript thoroughly. $\mathrm{MEB}$ and $\mathrm{BC}-\mathrm{B}$ provided the materials and funding to support this project.

\section{ACKNOWLEDGEMENTS}

We acknowledge and are grateful for our collaboration with Indiana University for performing the RNA sequencing.

\section{CONFLICTS OF INTEREST}

The authors declare no potential conflicts of interest.

\section{FUNDING}

This work was supported by grants from National Institute of Health, grants R01-CA125806-04 to MEB, 1R15CA176496-01A1 to MEB and R01-CA174785-A1 to BMC-B.

\section{REFERENCES}

1. Jhan JR, Andrechek ER. Triple-negative breast cancer and the potential for targeted therapy. Pharmacogenomics. 2017; 18:1595-609. https://doi.org/10.2217/pgs-2017-0117. PMID:29095114

2. Duffy MJ, McGowan PM, Crown J. Targeted therapy for triple-negative breast cancer: where are we? Int J Cancer. 2012; 131:2471-77. https://doi.org/10.1002/ijc.27632. PMID:22581656

3. Fleisher B, Clarke C, Ait-Oudhia S. Current advances in biomarkers for targeted therapy in triple-negative breast cancer. Breast Cancer (Dove Med Press). 2016; 8:183-97. https://doi.org/10.2147/BCTT.S114659 PMID:27785100
4. Low HB, Zhang Y. Regulatory roles of MAPK phosphatases in cancer. Immune Netw. 2016; 16:85-98. https://doi. org/10.4110/in.2016.16.2.85. PMID:27162525

5. Yang XL, Lin FJ, Guo YJ, Shao ZM, Ou ZL. Gemcitabine resistance in breast cancer cells regulated by PI3K/AKTmediated cellular proliferation exerts negative feedback via the MEK/MAPK and mTOR pathways. OncoTargets Ther. 2014; 7:1033-42. https://doi.org/10.2147/OTT.S63145. PMID:24966685

6. Grossi V, Peserico A, Tezil T, Simone C. p38 $\alpha$ MAPK pathway: a key factor in colorectal cancer therapy and chemoresistance. World J Gastroenterol. 2014; 20:9744-58. https://doi.org/10.3748/wjg.v20.i29.9744. PMID:25110412

7. Yeldag G, Rice A, Del Río Hernández A. Chemoresistance and the self-maintaining tumor microenvironment. Cancers (Basel). 2018; 10:471. https://doi.org/10.3390/ cancers 10120471. PMID:30487436

8. Hoang VT, Yan TJ, Cavanaugh JE, Flaherty PT, Beckman BS, Burow ME. Oncogenic signaling of MEK5-ERK5. Cancer Lett. 2017; 392:51-59. https://doi.org/10.1016/j. canlet.2017.01.034. PMID:28153789

9. Drew BA, Burow ME, Beckman BS. MEK5/ERK5 pathway: the first fifteen years. Biochim Biophys Acta Rev Cancer. 2012; 1825:37-48. https://doi.org/10.1016/j. bbcan.2011.10.002. PMID:22020294

10. Antoon JW, Martin EC, Lai R, Salvo VA, Tang Y, Nitzchke AM, Elliott S, Nam SY, Xiong W, Rhodes LV, CollinsBurow B, David O, Wang G, et al. MEK5/ERK5 signaling suppresses estrogen receptor expression and promotes hormone-independent tumorigenesis. PLoS One. 2013; 8:e69291. https://doi.org/10.1371/journal.pone.0069291. PMID:23950888

11. Cronan MR, Nakamura K, Johnson NL, Granger DA, Cuevas BD, Wang JG, Mackman N, Scott JE, Dohlman HG, Johnson GL. Defining MAP3 kinases required for MDAMB-231 cell tumor growth and metastasis. Oncogene. 2012; 31:3889-900. https://doi.org/10.1038/onc.2011.544. PMID:22139075

12. Da Silva L, Simpson PT, Smart CE, Cocciardi S, Waddell N, Lane A, Morrison BJ, Vargas AC, Healey S, Beesley J, Pakkiri P, Parry S, Kurniawan N, et al. HER3 and downstream pathways are involved in colonization of brain metastases from breast cancer. Breast Cancer Res. 2010; 12:R46. https://doi.org/10.1186/bcr2603. PMID:20604919

13. Terasawa K, Okazaki K, Nishida E. Regulation of c-Fos and Fra-1 by the MEK5-ERK5 pathway. Genes Cells. 2003; 8:263-73. https://doi.org/10.1046/j.13652443.2003.00631.x. PMID:12622723

14. Hoang VT, Matossian MD, Ucar DA, Elliott S, La J, Wright MK, Burks HE, Perles A, Hossain F, King CT, Browning VE, Bursavich J, Fang F, et al. ERK5 is required for tumor growth and maintenance through regulation of the extracellular matrix in triple negative breast cancer. 
Front Oncol. 2020; 10:1164. https://doi.org/10.3389/ fonc.2020.01164. PMID:32850332

15. Bakiri L, Macho-Maschler S, Custic I, Niemiec J, GuíoCarrión A, Hasenfuss SC, Eger A, Müller M, Beug H, Wagner EF. Fra-1/AP-1 induces EMT in mammary epithelial cells by modulating Zeb1/2 and TGF $\beta$ expression. Cell Death Differ. 2015; 22:336-50. https://doi.org/10.1038/ cdd.2014.157. PMID:25301070

16. van Staalduinen J, Baker D, Ten Dijke P, van Dam H. Epithelial-mesenchymal-transition-inducing transcription factors: new targets for tackling chemoresistance in cancer? Oncogene. 2018; 37:6195-211. https://doi.org/10.1038/ s41388-018-0378-x. PMID:30002444

17. Shibue $\mathrm{T}$ and Weinberg RA. EMT, CSCs, and drug resistance: the mechanistic link and clinical implications. 2017; 14:611-629. https://doi.org/10.1038/ nrclinonc.2017.44.

18. Scheel C, Weinberg RA. Cancer stem cells and epithelialmesenchymal transition: concepts and molecular links. Semin Cancer Biol. 2012; 22:396-403. https://doi. org/10.1016/j.semcancer.2012.04.001. PMID:22554795

19. Francou A, Andrerson KV. The epithelial-to-mesenchymal transition in development and cancer. Annu Rev Cancer Biol. 2020; 4:197-220. https://doi.org/10.1146/annurevcancerbio-030518-055425.

20. Dongre A, Weinberg RA. New insights into the mechanisms of epithelial-mesenchymal transition and implications for cancer. Nat Rev Mol Cell Biol. 2019; 20:69-84. https://doi. org/10.1038/s41580-018-0080-4. PMID:30459476

21. Zhou C, Nitschke AM, Xiong W, Zhang Q, Tang Y, Bloch M, Elliott S, Zhu Y, Bazzone L, Yu D, Weldon CB, Schiff R, McLachlan JA, et al. Proteomic analysis of tumor necrosis factor- $\alpha$ resistant human breast cancer cells reveals a MEK5/Erk5-mediated epithelial-mesenchymal transition phenotype. Breast Cancer Res. 2008; 10:R105. https://doi. org/10.1186/bcr2210. PMID:19087274

22. Pereira DM, Simões AE, Gomes SE, Castro RE, Carvalho T, Rodrigues CM, Borralho PM. MEK5/ERK5 signaling inhibition increases colon cancer cell sensitivity to 5-fluorouracil through a p53-dependent mechanism. Oncotarget. 2016; 7:34322-40. https://doi.org/10.18632/ oncotarget.9107. PMID:27144434

23. Ortiz-Ruiz MJ, Álvarez-Fernández S, Parrott T, Zaknoen S, Burrows FJ, Ocaña A, Pandiella A, Esparís-Ogando A. Therapeutic potential of ERK5 targeting in triple negative breast cancer. Oncotarget. 2014; 5:11308-18. https://doi. org/10.18632/oncotarget.2324. PMID:25350956

24. Miranda M, Rozali E, Khanna KK, Al-Ejeh F. MEK5ERK5 pathway associates with poor survival of breast cancer patients after systemic treatments. Oncoscience. 2015; 2:99-101. https://doi.org/10.18632/oncoscience.135. PMID:25859552

25. Simões AE, Rodrigues CM, Borralho PM. The MEK5/
ERK5 signalling pathway in cancer: a promising novel therapeutic target. Drug Discov Today. 2016; 21:165463. $\quad$ https://doi.org/10.1016/j.drudis.2016.06.010. PMID:27320690

26. Pereira DM, Rodrigues CM. Targeted avenues for cancer treatment: the MEK5-ERK5 signaling pathway. Trends Mol Med. 2020; 26:394-407. https://doi.org/10.1016/j. molmed.2020.01.006. PMID:32277933

27. Hess KR, Anderson K, Symmans WF, Valero V, Ibrahim N, Mejia JA, Booser D, Theriault RL, Buzdar AU, Dempsey PJ, Rouzier R, Sneige N, Ross JS, et al. Pharmacogenomic predictor of sensitivity to preoperative chemotherapy with paclitaxel and fluorouracil, doxorubicin, and cyclophosphamide in breast cancer. J Clin Oncol. 2006; 24:4236-44. https://doi.org/10.1200/JCO.2006.05.6861. PMID:16896004

28. Pavan S, Meyer-Schaller N, Diepenbruck M, Kalathur RK, Saxena M, Christofori G. A kinome-wide high-content siRNA screen identifies MEK5-ERK5 signaling as critical for breast cancer cell EMT and metastasis. Oncogene. 2018; 37:4197-213. https://doi.org/10.1038/s41388-018-0270-8. PMID:29713055

29. Shukla A, Miller JM, Cason C, Sayan M, MacPherson MB, Beuschel SL, Hillegass J, Vacek PM, Pass HI, Mossman BT. Extracellular signal-regulated kinase 5: a potential therapeutic target for malignant mesotheliomas. Clin Cancer Res. 2013; 19:2071-83. https://doi.org/10.1158/1078-0432. CCR-12-3202. PMID:23446998

30. Housman G, Byler S, Heerboth S, Lapinska K, Longacre M, Snyder N, Sarkar S. Drug resistance in cancer: an overview. Cancers (Basel). 2014; 6:1769-92. https://doi.org/10.3390/ cancers6031769. PMID:25198391

31. Weldon CB, Scandurro AB, Rolfe KW, Clayton JL, Elliott S, Butler NN, Melnik LI, Alam J, McLachlan JA, Jaffe $\mathrm{BM}$, Beckman BS, Burow ME. Identification of mitogenactivated protein kinase kinase as a chemoresistant pathway in MCF-7 cells by using gene expression microarray. Surgery. 2002; 132:293-301. https://doi.org/10.1067/ msy.2002.125389. PMID:12219026

32. Stecca B, Rovida E. Impact of ERK5 on the hallmarks of cancer. Int J Mol Sci. 2019; 20:1426. https://doi. org/10.3390/ijms20061426. PMID:30901834

33. Bhatt AB, Gupta M, Hoang VT, Chakrabarty S, Wright TD, Elliot S, Chopra IK, Monlish D, Anna K, Burow ME, Cavanaugh JE, Flaherty PT. Novel Diphenylamine Analogs Induce Mesenchymal to Epithelial Transition in Triple Negative Breast Cancer. Front Oncol. 2019; 9:672. https:// doi.org/10.3389/fonc.2019.00672. PMID:31417863

34. Chakrabarty S, Monlish DA, Gupta M, Wright TD, Hoang VT, Fedak M, Chopra I, Flaherty PT, Madura J, Mannepelli S, Burow ME, Cavanaugh JE. Structure activity relationships of anthranilic acid-based compounds on cellular and in vivo mitogen activated protein kinase-5 
signaling pathways. Bioorg Med Chem Lett. 2018; 28:2294-301. https://doi.org/10.1016/j.bmcl.2018.05.029. PMID:29803729

35. Lu X, Smaill JB, Ding K. New Promise and Opportunities for Allosteric Kinase Inhibitors. Angew Chem Int Ed. 2020; 59:13764-76. $\quad$ https://doi.org/10.1002/anie.201914525. PMID:31889388

36. Lu X, Smaill JB, Ding K. Medicinal Chemistry Strategies for the Development of Kinase Inhibitors Targeting Point Mutations. J Med Chem. 2020; 63:10726-41. https://doi. org/10.1021/acs.jmedchem.0c00507. PMID:32432477

37. Fabbro D, Cowan-Jacob SW, Moebitz H. Ten things you should know about protein kinases: IUPHAR Review 14. Br J Pharmacol. 2015; 172:2675-700. https://doi.org/10.1111/ bph.13096. PMID:25630872

38. Yudushkin I. Control of Akt activity and substrate phosphorylation in cells. IUBMB Life. 2020; 72:1115-25. https://doi.org/10.1002/iub.2264. PMID:32125765

39. Han B, Salituro FG, Blanco MJ. Impact of Allosteric Modulation in Drug Discovery: Innovation in Emerging Chemical Modalities. ACS Med Chem Lett. 2020; 11:1810 19. $\quad$ https://doi.org/10.1021/acsmedchemlett.9b00655. PMID:33062158

40. Du J, Guo J, Kang D, Li Z, Wang G, Wu J, Zhang Z, Fang H, Hou X, Huang Z, Li G, Lu X, Liu X, et al. New techniques and strategies in drug discovery. Chin Chem Lett. 2020; 31:1695-708. https://doi.org/10.1016/j. cclet.2020.03.028.

41. Liu F, Zhang H, Song H. Upregulation of MEK5 by Stat3 promotes breast cancer cell invasion and metastasis. Oncol Rep. 2017; 37:83-90. https://doi.org/10.3892/or.2016.5256. PMID:27878304

42. Brabletz T, Kalluri R, Nieto MA, Weinberg RA. EMT in cancer. Nat Rev Cancer. 2018; 18:128-34. https://doi. org/10.1038/nrc.2017.118. PMID:29326430

43. Bakir B, Chiarella AM, Pitarresi JR, Rustgi AK. EMT, MET, plasticity, and tumor metastasis. Trends Cell Biol. 2020; 30:764-76. https://doi.org/10.1016/j.tcb.2020.07.003. PMID:32800658

44. Imani S, Hosseinifard H, Cheng J, Wei C, Fu J. Prognostic value of EMT-inducing transcription factors (EMT-TFs) in metastatic breast cancer: A systematic review and metaanalysis. Sci Rep. 2016; 6:28587. https://doi.org/10.1038/ srep28587. PMID:27335258

45. Lehmann BD, Bauer JA, Chen X, Sanders ME, Chakravarthy AB, Shyr Y, Pietenpol JA. Identification of human triple-negative breast cancer subtypes and preclinical models for selection of targeted therapies. J Clin Invest. 2011; 121:2750-67. https://doi.org/10.1172/ JCI45014. PMID:21633166

46. Miller DF, Yan PS, Buechlein A, Rodriguez BA, Yilmaz AS, Goel S, Lin H, Collins-Burow B, Rhodes LV, Braun C, Pradeep S, Rupaimoole R, Dalkilic M, et al. A new method for stranded whole transcriptome RNA-seq. Methods. 2013; 63:126-34. https://doi.org/10.1016/j.ymeth.2013.03.023. PMID:23557989 